\title{
Optimized Readout Methods of Silicon Drift Detectors for High Resolution Spectroscopy in Micro-Beam Analysis
}

\author{
H.Soltau*, P.Lechner*, A. Niculae*, G. Lutz**, L. Strüder**, C. Fiorini*** and A. Longoni*** \\ R. Eckhard, ${ }^{*}$ G. Schaller, ${ }^{* *}$ and F. Schopper** \\ *PNSensor GmbH, Römerstr. 28, D-80803 München, Germany \\ **MPI Halbleiterlabor, Otto-Hahn-Ring 6, D-81739 München, Germany \\ ***Politecnico di Milano, Piazza L. da Vinci 32, I-20133 Milano, Italy
}

Silicon Drift Detectors (SDDs) fabricated by the Semiconductor Laboratory of the Max-PlanckInstitute in Munich in cooperation with PNSensor are widely used as X-ray sensors in XRF and Microbeam Analysis.

Minimization of the overall detector capacitance achieved by the sideward depletion scheme and a small area readout node, as well as the integration of the first amplification FET directly onto the detector (see Fig. 1) makes the SDD an excellent energy resolving device for micro-beam analysis applications. Some of the SDD features are:

- good energy resolution of 128 to $140 \mathrm{eV}$ for MnK-line

- high count rate capability up to $10^{6} \mathrm{cps}$

- good peak-to-background ratio of 2.500 up to 10.000

- a comfortable operation - all performance values are achieved with peltier cooling at operation temperatures between $-10^{\circ} \mathrm{C}$ and $-20^{\circ} \mathrm{C}$.

In the classical readout scheme of the SDD, the integrated transistor is operated in the sourcefollower configuration and the signal charge is removed continuosly by the detector self-reset mechanism. The advantages of this method are obvious: no detector dead time and only DC supply are required. The method gives very good results (see Fig. 2) at counting rates up to $10 \mathrm{kcps}$. For higher counting rates, the FWHM is increasing with the growing reset current. The change of the input capacitance leading to a peak shift is usually controlled by a feedback loop integrated into the readout circuit (peak shift stabilization).

One of the alternative readout schemes of the SDD is the so-called Charge-Sensitive-Amplifier (CSA) configuration. The signal charge collected at the readout node is integrated on a feedback capacitance across an inverting amplifier in which the integrated FET plays the role of the input transistor. The feedback capacitance can be either the parasitic capacitance between the transistor guard ring and the readout anode or an extra capacitance built on the SDD. The output signal depends only on the value of the feedback capacitor. This readout scheme offers the advantage of a very stable pulse height independent on the counting rate.

In applications requiring very high counting rates and constant energy resolution over the whole counting rate range, pulsed-reset operation of the SDD is desirable. This is achieved by applying reset pulses to a reset diode integrated on the readout anode on the chip. The combination of the CSA readout scheme and the pulsed-reset method insures very good peak stability and energy resolution independent on the count rate. It provides a pulsed ramp output signal to be used with conventional and commercially available analog and digital electronics.

We will present the different readout methods of the SDD, draw attention to their specific applications and report on selected measurement results. 


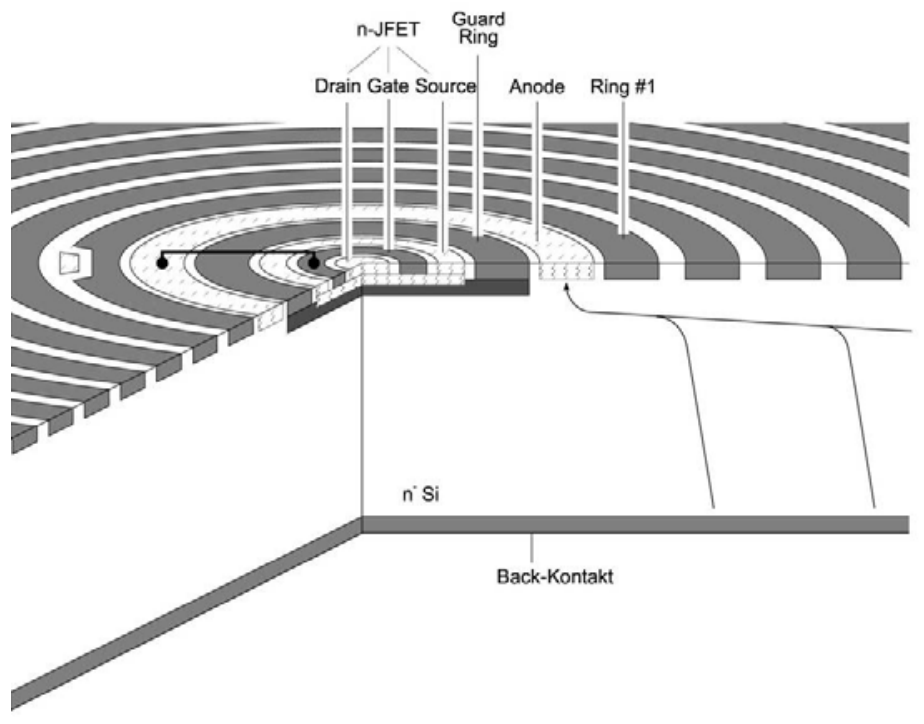

Fig. 1 Silicon Drift Detector (SDDs) marked by their integrated first amplification step are providing high energy resolution and count rate capability at comfortable operation conditions.

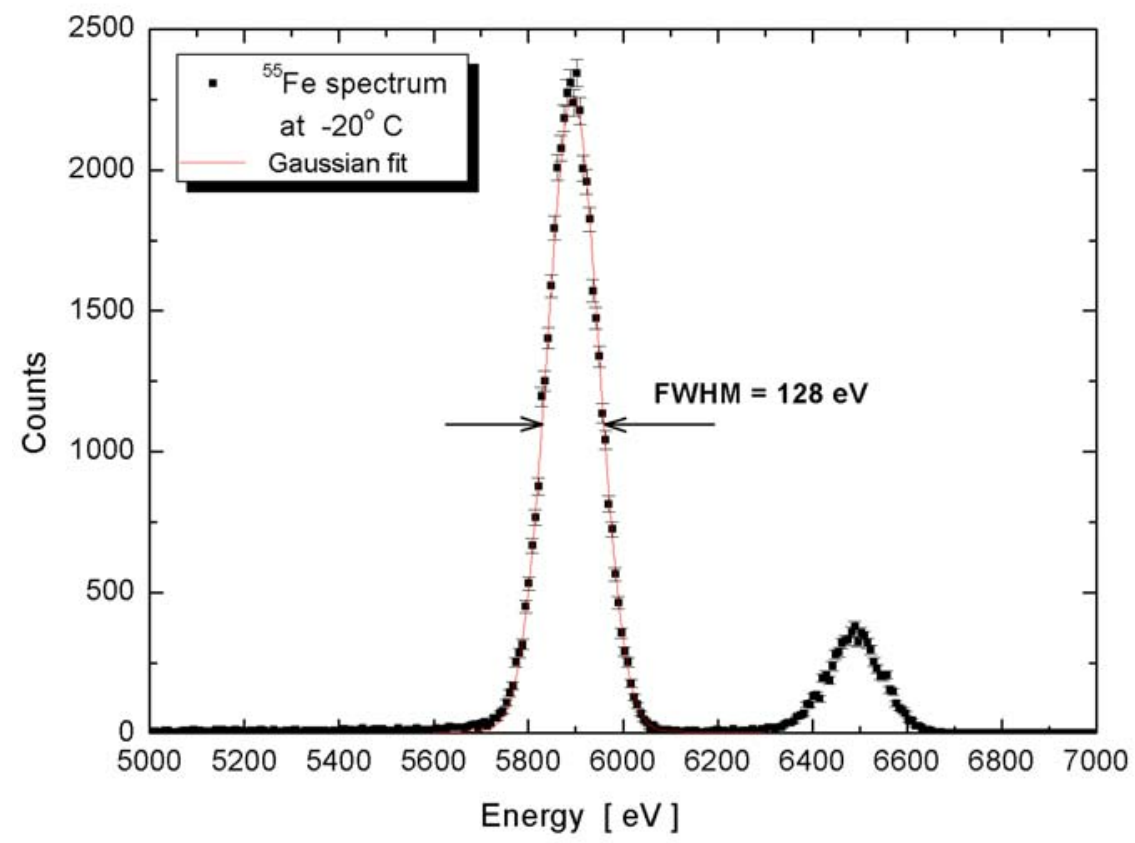

Fig. 2 X-ray spectrum of the radioactive source ${ }^{55} \mathrm{Fe}$ at $-20^{\circ} \mathrm{C}$ from a $10 \mathrm{~mm}^{2} \mathrm{SDD}$ of the high resolution type with $1 \mu$ s shaping time and $2000 \mathrm{cps}$. 